University of South Carolina

Scholar Commons

6-1978

\title{
Bayes Estimation of Reliability for Mixtures of Life Distributions
}

William J. Padgett

University of South Carolina - Columbia, padgettw@bellsouth.net

Chris P. Tsokos

Follow this and additional works at: https://scholarcommons.sc.edu/math_facpub

Part of the Mathematics Commons, and the Statistics and Probability Commons

\section{Publication Info}

Siam Journal on Applied Mathematics, Volume 34, Issue 4, 1978, pages 692-703.

(c) 1978 by Society for Industrial and Applied Mathematics

This Article is brought to you by the Mathematics, Department of at Scholar Commons. It has been accepted for inclusion in Faculty Publications by an authorized administrator of Scholar Commons. For more information, please contact digres@mailbox.sc.edu. 


\title{
ON BAYES ESTIMATION OF RELIABILITY FOR MIXTURES OF LIFE DISTRIBUTIONS*
}

\author{
W. J. PADGETT $\dagger$ AND C. P. TSOKOS $\ddagger$
}

\begin{abstract}
Assuming that the conditional failure time distribution $F(t \mid \theta)$ of a piece of equipment depends on a random parameter with probability distribution function $G(\theta)$, the unconditional failure time distribution is given by $F(t)=\int F(t \mid \theta) d G(\theta)$. Utilizing the ideas of Dirichlet process priors on $F(t)$, Bayes estimators of the unconditional reliability function $R(t)=1-F(t)$ are discussed. The robustness of the Bayes estimators with respect to the choice of $F(t \mid \theta)$ and $G(\theta)$ is studied by Monte Carlo simulation. In particular, the Weibull, lognormal, and gamma distributions are considered for $F(t \mid \theta)$ and the inverted gamma and "general" uniform distributions are used for $G(\theta)$, where $\theta$ is assumed to be a random scale parameter.
\end{abstract}

1. Introduction. Mathematically, the description of the length of life of a piece of equipment is assumed to be given by a failure time (or life) distribution. That is, a probability distribution of a certain form is usually assumed to give the reliability of a piece of equipment for each time $t$. However, since there are many physical causes that individually or collectively may be responsible for the failure of a device at any given instant, and since it is not generally possible to isolate these causes, the choice of a failure distribution is still an art. The selection of the failure distribution is thus subject to uncertainty.

A typical failure distribution that may be chosen will depend on at least one unknown parameter. In many situations, the parameter involved must be thought of as a value of a random variable which behaves in accordance with an unknown probability distribution. For instance, if items are manufactured under conditions which, even though carefully controlled, cause the parameter of the failure distribution of the items to change randomly from one item to another, then this situation arises. Also, if several lots of manufactured devices are mixed in a bin and the parameter of the failure distribution changes randomly from one lot to another, then a randomly chosen item from the bin has a failure distribution function depending on the value of a random parameter. In any case, the probability distribution of the random parameter must either be assumed or approximated if enough data are available in order to obtain an expression for the reliability of a device. Again, the selection of the probability distribution of the random parameter is subject to uncertainty.

Notation and basic definitions. Let $F(t \mid \theta), t \in R$, be a failure time distribution function depending on a parameter $\theta$ which belongs to some set $\Omega \subset R$, where $R$ denotes the real line. Suppose the exact form of $F(t \mid \theta)$ is not known, but using goodness-of-fit procedures on pilot life data or data on similar items in an allied product line, the engineer can obtain an initial guess of the type of distribution involved. For example, it may be assumed that $F(t \mid \theta)$ is a Weibull distribution function with unknown scale parameter $\theta$. In addition, we suppose that $\theta$ is a value of a random variable $\Theta$ which has a distribution function denoted by $G(\theta), \theta \in \Omega$. Again, an initial guess at the distribution $G(\theta)$ can be made using prior information about the items or components involved. For example, it may be assumed in certain cases that $G(\theta)$ is a uniform or an inverted gamma distribution function over $\Omega$.

* Received by the editors November 18,1976 , and in final revised form June 15,1977 . This work was supported by the United States Air Force Office of Scientific Research under Grant AFOSR-74-2711.

$\dagger$ Department of Mathematics and Computer Science, University of South Carolina, Columbia, South Carolina 29208.

$\ddagger$ Department of Mathematics, University of South Florida, Tampa, Florida 33620. 
The failure time distribution of mixed devices (as in the bin of items mentioned earlier) is a mixture of the distributions $F(t \mid \theta)$ with mixing distribution $G(\theta)$. Thus, the unconditional failure distribution function $F(t)$ is given by

$$
F(t)=\int_{\Omega} F(t \mid \theta) d G(\theta)
$$

$t \in R$.

The distribution function (1.1) is a compound distribution and is interpreted as the failure time distribution of an item selected randomly from a bin of items with $\theta$ varying randomly from one lot (or item) to another. Thus, given a random sample of lifetimes of items generated from a distribution such as (1.1), we desire an estimate of the unconditional reliability function at each $t$,

$$
R(t)=1-F(t)
$$

$t \in R$,

based on the sample values.

If an initial guess of either $F(t \mid \theta)$ or $G(\theta)$ or both are used in (1.1), we will denote the initial guess of $F(t)$ by $F_{0}(t)$ in later sections.

Objectives. Harris and Singpurwalla [5] considered estimation of the reliability function $R(t)$ when the exact form of the failure distribution $F(t \mid \theta)$ and the distribution $G(\theta)$ were known. Their estimate involved only the estimation of the parameters of $G(\theta)$ and other parameters of $F(t \mid \theta)$. However, if the engineer is uncertain of the particular forms of $F(t \mid \theta)$ and $G(\theta)$, then he may have chosen the wrong probability models in the first place and should not put very much weight on these initial guesses.

The types of problems mentioned here are well suited to the techniques of Bayes estimation of distribution functions obtained by Ferguson [3], [4]. Ferguson considered Bayes estimation of an unknown distribution function with respect to a Dirichlet process as a prior distribution on the class of all distribution functions over the real line. In this paper, we consider the Bayes estimation of the unconditional reliability function $R(t)$ utilizing the Dirichlet process as a prior distribution. The Dirichlet process prior will be given in terms of the initial guess $F_{0}$ where either $F(t \mid \theta)$ or $G(\theta)$ (or both) must be assumed or estimated from prior data to obtain $F_{0}$.

We first give a brief discussion of the Dirichlet process. Then the Bayes estimation of reliability utilizing the Dirichlet process is considered in $\S 3$. Finally, the robustness of the Bayes estimator of $R(t)$ with respect to the initial guesses at $F(t \mid \theta)$ and $G(\theta)$ is studied by Monte Carlo simulations. Specifically, the Weibull distribution, gamma distribution, and lognormal distribution are considered for $F(t \mid \theta)$, where $\theta$ is the scale parameter, and the inverted gamma and "general uniform" distributions are used for $G(\theta)$, since these families provide a rich class of mixing distributions with respect to the scale parameter.

2. The Dirichlet process. In this section we will define what is meant by a Dirichlet process and state some of its useful properties as given by Ferguson [3].

First, we recall that a $k$-dimensional random vector has a Dirichlet distribution with parameter $\left(\nu_{1}, \cdots, \nu_{k+1}\right)$ if its probability density function is given by (Wilks [9, p. 177])

$$
\begin{aligned}
f\left(x_{1}, \cdots, x_{k} ; x_{k+1}\right)= & \frac{\Gamma\left(\nu_{1}+\cdots+\nu_{k+1}\right)}{\prod_{i=1}^{k+1} \Gamma\left(\nu_{i}\right)} x_{1}^{\nu_{1}-1} \cdots x_{k}^{\nu_{k}-1} x_{k^{k+1}}^{\nu^{k}-1} \\
& \text { for } x_{i} \geqq 0, \quad i=1, \cdots, k \text { and } \sum_{i=1}^{k} x_{i} \leqq 1, \quad x_{k+1}=1-\sum_{i=1}^{k} x_{i} .
\end{aligned}
$$

Here $\Gamma(\nu)$ denotes the usual gamma function. 
Definition. Let $\alpha(\cdot)$ be a finite nonnull measure on $(R, \mathscr{B})$, where $\mathscr{B}$ denotes the Borel subsets of $R$, and let $P(\cdot)$ denote a stochastic process indexed by elements of $\mathscr{B}$. We say that $P$ is a Dirichlet process with parameter $\alpha$, and write $P \in \mathscr{D}(\alpha)$, if for every finite measurable partition $\left\{B_{1}, \cdots, B_{n}\right\}$ of $R$ (that is, $B_{i}$ is an element of $\mathscr{B}, B_{i}$ and $B_{j}$ are disjoint for $i \neq j$, and $\left.\cup_{i=1}^{n} B_{i}=R\right),\left(P\left(B_{1}\right), \cdots, P\left(B_{n}\right)\right)$ has the Dirichlet distribution with parameter $\left(\alpha\left(B_{1}\right), \cdots, \alpha\left(B_{n}\right)\right)$.

Note that $P$ is simply a random probability measure on the Borel subsets of $R$. We let $F(t)=P((-\infty, t]), t \in R$, denote the corresponding distribution function. Then we say $F \in \mathscr{D}(\alpha)$ to mean $P \in \mathscr{D}(\alpha)$. We also denote $\alpha(t)=\alpha((-\infty, t])$.

Some properties of Dirichlet processes are listed next.

Property 1. If $F \in \mathscr{D}(\alpha)$, then $F$ is discrete with probability one.

Property 2. For any nonnegative measurable function $g, \int_{-\infty}^{\infty} g(t) d \alpha(t)<\infty$ if and only if $\int_{-\infty}^{\infty} g(t) d F(t)<\infty$ with probability one.

Property 3. For every $B \in \mathscr{B}, P(B)$ has the beta distribution (one-dimensional Dirichlet distribution) with parameter $(\alpha(B), \alpha(R)-\alpha(B))$. Hence, $E[P(B)]=$ $\alpha(B) / \alpha(R)$.

Property 4. If $F \in \mathscr{D}(\alpha)$ and $X_{1}, \cdots, X_{n}$ is a random sample from $F$, then the posterior distribution of $F$, given $X_{1}, \cdots, X_{n}$, is a Dirichlet process with parameter $\alpha+\sum_{i=1}^{n} \delta_{X_{i}}$, where $\delta_{x}(B)=1$ if $x \in B$ and $\delta_{x}(B)=0$ if $x \notin B$.

Properties 3 and 4 are extremely useful for the estimation of $R(t)$. Also, Property 2 indicates a relationship between the parameter $\alpha$ of the Dirichlet process and the distribution function $F$.

3. Bayes estimation of unconditional reliability. We now consider the Bayes estimation of the unconditional reliability function $R(t)=1-F(t), t \in R$, where $F(t)$ is given by (1.1).

Let $X_{1}, \cdots, X_{n}$ denote a random sample of failure times from the unconditional failure distribution $F(t)$ given by (1.1). We wish to estimate $F$ by a probability distribution function $\hat{F}$ with respect to the loss function

$$
L(F, \hat{F})=\int_{-\infty}^{\infty}[F(t)-\hat{F}(t)]^{2} d W(t),
$$

where $W(t)$ is some given (nonrandom) weight function. Following Ferguson [3], [4], as a prior distribution on $F$, we take $F \in \mathscr{D}(\alpha)$, that is, $F$ is a Dirichlet process with parameter $\alpha$. If no data were observed, since by Property 3 we always have

$$
F(t)=P((-\infty, t]) \sim \operatorname{Beta}(\alpha(t), \alpha(R)-\alpha(t)),
$$

the Bayes estimate of $F(t)$ for the no-data case is the mean of the posterior (given no data) distribution, which is

$$
F_{0}(t)=E[F(t)]=\frac{\alpha(t)}{\alpha(R)} .
$$

Hence, we can take $F_{0}(t)=\alpha(t) / \alpha(R)$ as the prior guess of $F(t)$ since no data were observed. The prior guess is thus strictly determined by $\alpha$. Therefore, when the prior guess of $F(t)$ is specified as $F_{0}(t)$, the parameter $\alpha$ of the Dirichlet process is being specified. Also, $F_{0}(t)$ is specified if either $F(t \mid \theta)$ or $G(\theta)$ are estimated or chosen based on prior knowledge or prior data.

Now, given the sample data $X_{1}, \cdots, X_{n}$, by Property 4 , the posterior distribution of $F$ is a Dirichlet process with parameter $\alpha+\sum_{i=1}^{n} \delta_{X_{i}}$. Hence, again using Property 3 , the Bayes estimate of $F(t)$ is found to be (see [3]) the mean of the posterior distribution 
of $F$, given $X_{1}, \cdots, X_{n}$, that is,

$$
\begin{aligned}
\hat{F}_{n}(t) & =E\left[F(t) \mid X_{1}, \cdots, X_{n}\right] \\
& =\frac{\left(\alpha+\sum_{i=1}^{n} \delta_{X_{i}}\right)((-\infty, t])}{\left(\alpha+\sum_{i=1}^{n} \delta_{X_{i}}\right)(R)} \\
& =\frac{\alpha(t)+\sum_{i=1}^{n} I_{\left[X_{i} \infty\right)}(t)}{\alpha(R)+n},
\end{aligned}
$$

where $I_{A}(t)=1$ if $t \in A$ and $I_{A}(t)=0$ otherwise. If we let $F_{n}(t)$ denote the sample distribution function and

$$
p_{n}=\frac{\alpha(R)}{\alpha(R)+n}
$$

then (3.1) may be written as

$$
\hat{F}_{n}(t)=p_{n} F_{0}(t)+\left(1-p_{n}\right) F_{n}(t) .
$$

Thus, the Bayes estimate of $F(t)$ with respect to the "squared error" loss function $L(F, \hat{F})$ and the Dirichlet process prior is a mixture of the prior guess at $F$ and the observed sample distribution function.

Note that since $\alpha$ is a finite measure and $p_{n} \rightarrow 0$ as $n \rightarrow \infty$, we have for the sequence of estimators the desirable property that $\hat{F}_{n}(t) \rightarrow F(t)$ almost surely uniformly in $t$ since $F_{n}(t) \rightarrow F(t)$ almost surely uniformly in $t$ by the Glivenko-Cantelli theorem.

Point estimation of reliability. For the reliability estimation problem at hand, we take the measure $\alpha$ to be

$$
\alpha(\dot{A})=Q(R) \int_{-\infty}^{\infty} P(A \mid \theta) d G(\theta), \quad A \in \mathscr{B},
$$

where $P(\cdot \mid \theta)$ is the probability measure on $R$ corresponding to the assumed (or initial guess of the) conditional failure distribution function $F(t \mid \theta), G(\theta)$ is the assumed (or initial guess of the) distribution of $\Theta$, and $Q(\cdot)$ is some finite nonnull measure on $(R, \mathscr{B})$. Then $\alpha(R)=Q(R)$ since $P(R \mid \theta)=1$, and taking $A=(-\infty, t]$, we have from (3.2) that

$$
\alpha(t)=Q(R) \int_{-\infty}^{\infty} F(t \mid \theta) d G(\theta)
$$

Hence,

$$
F_{0}(t)=\alpha(t) / Q(R)=\int_{-\infty}^{\infty} F(t \mid \theta) d G(\theta)
$$

and

$$
p_{n}=\frac{Q(R)}{Q(R)+n} .
$$

Therefore, based on a random sample $X_{1}, \cdots, X_{n}$ from $F(t)$, the Bayes estimate of the unconditional reliability function is from (3.1)

$$
\hat{R}_{n}(t)=1-\frac{Q(R)}{Q(R)+n} F_{0}(t)-\frac{1}{Q(R)+n} \sum_{i=1}^{n} I_{\left[X_{i}, \infty\right)}(t) .
$$


Note that the value $Q(R)$ in (3.4) may be considered as a measure of the "faith" that is placed in the prior guess of $F$ since $p_{n}$ is the weight placed on $F_{0}$. Actually, this is the only way in which $Q$ enters the estimator, since the initial guess of $F$ from (3.3) depends only on the prior guess of $F(t \mid \theta)$ or $G(\theta)$ or both and not on $Q$. The value of $Q(R)$ may be chosen according to the amount of prior information available to the experimenter when making his choice of $F_{0}(t)$. A large amount of prior information may indicate a large value of $Q(R)$. Hence, $Q(R)$ is a function of the amount of prior information available for choosing $F_{0}$, but is not necessarily equal to the number of prior observations.

We now give the form of $F_{0}$ (or more specifically, its density function) for several assumed failure distributions $F(t \mid \theta)$ and prior distributions $G(\theta)$ when $\theta$ is an unknown scale parameter.

First, we consider a general class of failure distributions $F(t \mid \theta)$ with density function of the form

$$
f(t \mid \theta)=g^{\prime}(t ; \theta) e^{-g(t ; \theta)}, \quad t \in S \subset R,
$$

where $g(t ; \theta)$ is a nonnegative function such that $e^{-g(t ; \theta)} \rightarrow 0$ as $t \rightarrow \infty$ for each $\theta$. Then it is obvious that special cases of this class of failure distributions are the exponential, Weibull, and extreme value distributions.

Suppose that $G(\theta)$ is the uniform distribution on an interval $(a, b)$ and take $g(t ; \theta)=g(t) \theta$, where $g(t)$ is nonnegative and $g^{\prime}(t)$ exists and is continuous on the interior of $S$. Then the initial guess (or prior guess) of the distribution $F(t)$ is the distribution function $F_{0}(t)$ which has a probability density function given by

$$
\begin{aligned}
f_{0}(t) & =\int_{-\infty}^{\infty} f(t \mid \theta) d G(\theta) \\
& =\int_{a}^{b} g^{\prime}(t) \theta e^{-g(t) \theta} \frac{1}{b-a} d \theta \\
& =\frac{g^{\prime}(t)}{b-a}\left[\frac{a e^{-a g(t)}-b e^{-b g(t)}}{g(t)}+\frac{e^{-a g(t)}-e^{-b g(t)}}{g^{2}(t)}\right], \quad t \in S .
\end{aligned}
$$

Here we have taken $Q(R)=1$ so $\alpha(t)=F_{0}(t)$. In fact, we may take $Q(R)$ to be any constant which reflects how strongly we feel about our choice for $f_{0}$. We choose $Q(R)=1$ only for illustration at this point. Thus, the initial or prior guess of $F(t)$ for this case is the distribution function

$$
F_{0}(t)=\int_{-\infty} f_{0}(u) d u=1+\frac{e^{-b g(t)}-e^{-a g(t)}}{g(t)(b-a)}, \quad t \in S,
$$

which is then used in (3.4) to obtain the Bayes estimator of $R(t)$. Note that in this case if we change variables from $\theta$ to $\eta=1 / \theta$, then $g(t ; \eta)=g(t) / \eta$ and $\eta$ is a value of a random variable which has density function given by one of the "general uniform" densities of Tsokos [8], that is, the density corresponding to the distribution $G(\eta)$ is

$$
h(\eta)= \begin{cases}\frac{1}{\eta^{2}(b-a)}, & \frac{1}{b}<\eta<\frac{1}{a}, \\ 0, & \text { otherwise }\end{cases}
$$

Next, for the general class of conditional failure distributions (3.5), we take 
$g(t ; \theta)=g(t) / \theta$ and assume that $G(\theta)$ is the inverted gamma distribution with parameters $(\mu, \nu)$. That is, a density function for $G$ is

$$
\gamma(\theta)=\frac{1}{\mu \Gamma(\nu)}\left(\frac{\mu}{\theta}\right)^{\nu+1} e^{-\mu / \theta}, \quad \theta>0 \quad(\mu, \nu>0) .
$$

Thus, the prior guess $F_{0}(t)$ has a density function given by

$$
\begin{aligned}
f_{0}(t) & =\int_{0}^{\infty} \frac{g^{\prime}(t)}{\theta} e^{-g(t) / \theta} \frac{1}{\mu \Gamma(\nu)}\left(\frac{\mu}{\theta}\right)^{\nu+1} e^{-\mu / \theta} d \theta \\
& =\frac{\nu g^{\prime}(t)}{\mu}\left[\frac{\mu}{g(t)+\mu}\right]^{\nu+1}, \quad t \in S .
\end{aligned}
$$

Hence, the distribution function $F_{0}(t)$ is

$$
F_{0}(t)=1-\left[\frac{\mu}{g(t)+\mu}\right]^{\nu}
$$

By taking $g(t ; \theta)=\theta t, t>0$, we obtain the exponential distribution from (3.5), and (3.6)-(3.7) are modified accordingly. Also, by taking $g(t ; \theta)=t^{\xi} / \theta, t>0$, we have from (3.5) the Weibull distribution with two parameters, and if $g(t ; \theta)=e^{\theta t},-\infty<t<\infty$, we obtain the extreme-value or Gumbel distribution.

Other useful failure models for reliability problems are the gamma distribution with parameters $\left(\alpha^{*}, \beta\right)$ and the lognormal distribution with parameters $\left(\lambda, \sigma^{2}\right)$. Table 1 gives the corresponding density functions $f_{0}(t)$ for these two distributions for either the uniform or inverted gamma prior distributions on the scale parameters. In Table 1, $\gamma(m, x)=\int_{0}^{x} u^{m-1} e^{-u} d u$ is the incomplete gamma function.

TABLE 1

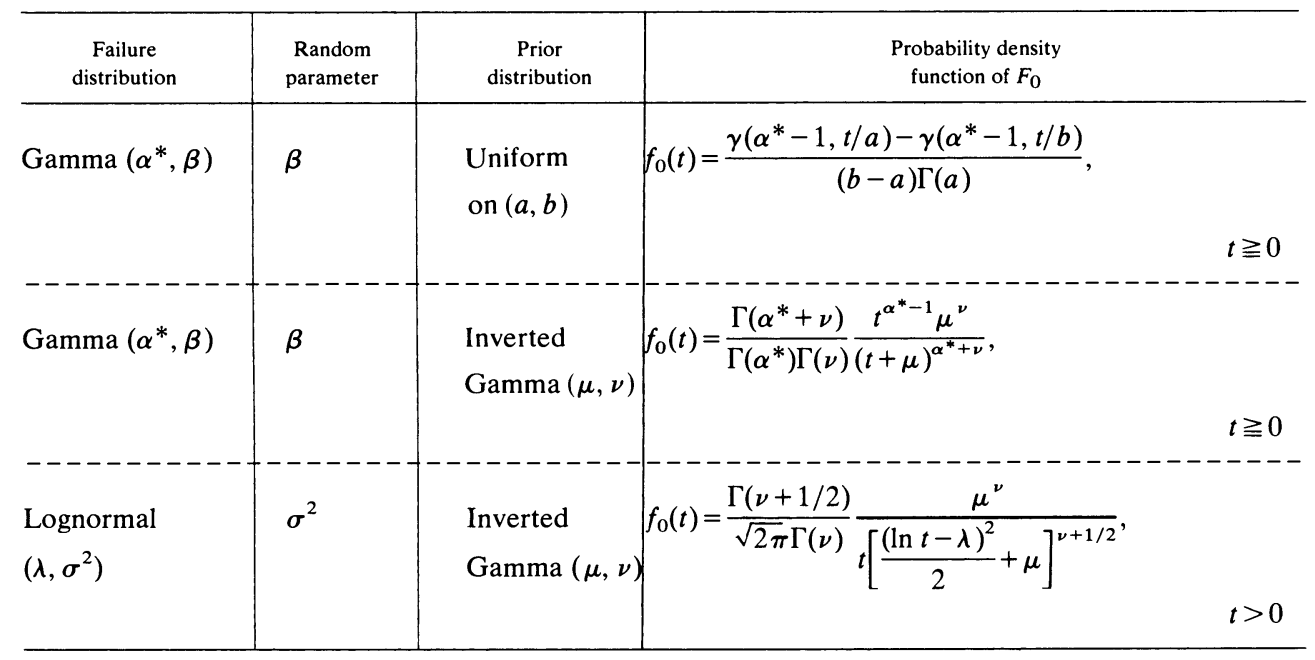

Bayesian interval estimation of reliability. Now, we will briefly discuss the problem of obtaining a Bayesian interval estimate of $R(t)$ for fixed $t>0$ using the Dirichlet process as a prior for $F$.

By Properties 3 and 4 of Dirichlet processes, for $t$ fixed the posterior distribution of $F(t)$, given the sample $X_{1}, \cdots, X_{n}$, is a beta distribution with parameters $\left(A_{n}(t), B_{n}(t)\right)$, 
where

$$
A_{n}(t)=\alpha(t)+\sum_{i=1}^{n} I_{\left[X_{i}, \infty\right)}(t)
$$

and

$$
B_{n}(t)=\alpha(R)+n-A_{n}(t) .
$$

Thus, since the posterior distribution of $R(t)=1-F(t)$, given $X_{1}, \cdots, X_{n}$, is the beta distribution with parameters $\left(B_{n}(t), A_{n}(t)\right)$, we may find an interval $(L, U)$, where $L$ and $U$ are two numbers such that for a given $0<\gamma<1$,

$$
\begin{aligned}
P\left[L<R(t)<U \mid X_{1}, \cdots, X_{n}\right] & =I_{U}\left(B_{n}(t), A_{n}(t)\right)-I_{L}\left(B_{n}(t), A_{n}(t)\right) \\
& =\gamma, \quad t>0 \text { fixed, }
\end{aligned}
$$

where $I_{x}(u, v)$ denotes the incomplete beta function ratio. Note that the interval depends on the initial guess of $F(t)$ through $\alpha(t)$.

Once the data are observed, it is not difficult to obtain the values of $L$ and $U$ that give (3.8) and so that the length of the interval $(L, U)$ is shortest. Equivalently, we may find $(L, U)$ that gives the highest probability density (HPD) interval of Box and Tiao [1, Chap. 2]. The tables of Pearson Type I (or II) percentage points (Pearson and Hartley [7]) may be used to do this, at least approximately.

4. A robustness study of the Bayes estimator. In this section using Monte Carlo simulation we consider the robustness of the Bayes estimator (3.4) with respect to the initial or prior guess $F_{0}$. Since $F_{0}$ depends on the prior guess of the conditional failure distribution $F(t \mid \theta)$ and on the choice of the distribution $G(\theta)$ of the random parameter, we study the behavior of the Bayes estimate $\hat{R}_{n}(t)$ with respect to the particular distributions $F_{0}$ given by (3.6), (3.7), and Table 1 , where $G(\theta)$ is an inverted gamma distribution or a general uniform distribution. At the same time the simulations will indicate some of the small sample properties of the estimator.

For a fixed value of $n$, a random sample of size $n$ was generated from the true unconditional failure distribution $F(t)$. Then an estimate of $R(t)$ was obtained from (3.4) where the prior guess $F_{0}(t)$ was found from (3.3) using the true conditional failure distribution $F(t \mid \theta)$. An estimate of $R(t)$ was also calculated from (3.4) using the assumed conditional failure distribution in (3.3) to obtain $F_{0}(t)$. The squared error of each estimate was then computed and stored. The entire procedure was repeated for 5,000 random samples of size $n$ at several different values of $t$. The estimated mean and variance as well as the estimated mean squared error were computed from the 5,000 values of each estimator. The entire simulation was repeated for several choices of $n$. The simulation was also performed in several cases using 10,000 random samples, but the results did not differ appreciably from the simulation using 5,000 samples.

In matching the true and assumed conditional failure distributions for the robustness study, values of the appropriate shape parameters were selected to give the same general shape and then the moments were matched when possible. Also, the conditional failure distributions were matched by modes.

In Tables 2-6 some of the results are presented which are representative of the Monte Carlo study. To obtain Table 2, for example, the true conditional lifetime (or failure) distribution was lognormal with mean and variance of the logarithm of the lifetime equal to $m$ and $\sigma^{2}$, respectively, whereas the assumed conditional lifetime distribution was Weibull with shape parameter $\xi$ and scale parameter $\theta$. The distribution $G$ of the random scale parameter $\sigma^{2}$ (or $\theta$ ) was inverted gamma with parameters 
$(\mu, \nu)$ as given in $\S 3$. Taking $\xi=2$ gives a unimodal Weibull lifetime distribution, and $\mu=2$ and $\nu=5$ give the mean of the distribution of the scale parameter $\sigma^{2}$ (or $\theta$ ) equal to $\mu /(\nu-1)=0.5$ and variance $\mu^{2} /\left((\nu-1)^{2}(\nu-2)\right)=0.0833$. Matching moments of the lognormal life distribution and the Weibull distribution with $\xi=2$, we have $m=-1.06$ (where $\sigma^{2}$ and $\theta$ have been replaced by the expected value 0.5 ). Matching modes and taking $\mu=8$ and $\nu=3$ in the inverted gamma distribution for the scale parameters, for example, we have for $\xi=2$ that $m=5.04$. Several other values of $\mu$ and $\nu$ were used giving results similar to those shown in Table 2 . Notice that when the conditional life distributions have matched moments the estimated mean squared error ratio is near one, indicating close agreement of the estimators. However, if the conditional life distributions have equal modes, the estimates are quite different. This was true for $n=10$ also, even though, as was mentioned in $\S 3$, the estimator $\hat{R}_{n}(t)$ converges almost surely to $R(t)$ uniformly in $t$ as $n \rightarrow \infty$. The large difference in MSE ratio in Tables 2 and 3 could be due to the heavier tail of the lognormal distribution as compared to the gamma or Weibull distributions that are used, since in Table 4 the difference is not quite as pronounced. This reasoning seems to be valid since the MSE ratio is closer to one for small values of $t$, but decreases for large $t$ values in Tables 2 and 3 .

It should be remarked that the estimated variances are equal for both estimators in all tables since the only difference in the estimators enters through the nonrandom term $F_{0}(t)$ in (3.4). Also, the value $Q(R)=1$ was used for the results in Tables $1-5$, indicating little "faith" in the prior guesses at $F_{0}$ relative to the current observed sample of size $n$. Other values of $Q(R)$ may be used as will be discussed later in connection with Table 6 .

Note that the sample size in Tables 3 and 4 is $n=10$. The results for matched moments do not appear in Table 4 since this requires both $\xi$ and $\alpha^{*}$ (the shape parameter of the gamma distribution) to be one, and thus, we obtain exactly the same conditional life distribution in this case.

Now, with respect to the distribution $G$ of the scale parameters, Table 5 indicates that even for samples of size 5 with $Q(R)=1$ the estimators of $R(t)$ for the Weibull life distribution $F(t \mid \theta)$ are fairly robust. Also, in Table 6 the value of $Q(R)$ is 10 , which weights the prior guess and the sample distribution function equally when $n=10$. In this case also the estimators are fairly robust. The parameters $\mu$ and $\nu$ for the inverted gamma distribution $G(\theta)$ in Tables 5-6 were chosen similar to the values used by Canavos and Tsokos [2]. The alternative form of $G$ was chosen to be the "general uniform" of Tsokos [8] with density as given in $\S 3$ by

$$
g(\theta)= \begin{cases}\frac{1}{\theta^{2}(b-a)}, & \frac{1}{b}<\theta<\frac{1}{a} \\ 0, & \text { otherwise }\end{cases}
$$

Hence, matching the moments of the inverted gamma distribution with $\mu=40$ and $\nu=3$, for example, and the "general uniform" distribution above gives $a=0.008$ and $b=0.158$, approximately. This choice of distributions for $G$ seems appropriate in view of the recent conclusion of Higgins and Tsokos [6] that the inverted gamma and uniform distributions may both adequately fit a set of data from the Weibull lifetime distribution with a random scale parameter.

In general, the simulation study indicated that the estimator (3.4) is quite robust with respect to the choice of $F_{0}$ in some situations when the true and assumed conditional failure time distributions have the same general shape and same first and 
second moments. However, if the conditional failure time distributions have the same shape and equal modes, the estimator is sensitive to departure from the true failure time distribution. With respect to the choice of the distribution $G$ of the scale parameter, where $G$ is either a "general uniform" or an inverted gamma distribution, however, the estimator of $R(t)$ was quite robust. This is due to the fact that either of those distributions used for $G$ may well fit data which actually come from the other when the first two moments are the same. However, since the first few moments may usually be estimated (or assumed) from previous data in order to obtain a guess at $G$, it seems reasonable to consider such pairs of distributions for comparison. If this fitting property of the pair of distributions were not present, then of course the behavior of the Bayes estimator $\hat{R}_{n}(t)$ would be different, at least for small sample size $n$. In any case, if $Q(R)$ is chosen to be very large relative to $n$, then more faith is being placed on the wrong choice for $F_{0}$, and the estimator does not perform very well. For example, when $n=10$ a value of $Q(R)=100$ was tried in the cases given in Table 6 with very poor results (the MSE ratio when $\xi=1$ ranged from 0.787 at $t=0.4$ to 0.244 at $t=5.2$ ).

TABLE 2

Robustness of Bayes reliability estimator: True life distribution-lognormal $\left(m, \sigma^{2}\right)$, assumed life distribution-Weibull $(\xi, \theta)$, and inverted gamma $(\mu, \nu)$ prior distribution on scale parameter.

Sample size $=5, Q(R)=1$.

\begin{tabular}{l|c|c|c|c|c|c}
\hline \multicolumn{7}{c}{ Matching moments of life distributions $(m=-1.06, \xi=2, \mu=2, \nu=5)$} \\
\hline \multirow{3}{*}{ Tıme } & $\begin{array}{c}\text { Life } \\
\text { Distribution }\end{array}$ & $\begin{array}{l}\text { Ave. True } \\
\text { Reliability }\end{array}$ & $\begin{array}{c}\text { Estimated } \\
\text { Mean Rel. }\end{array}$ & $\begin{array}{c}\text { Estimated } \\
\text { Variance }\end{array}$ & $\begin{array}{c}\text { Estimated } \\
\text { MSE }\end{array}$ & $\begin{array}{c}\text { MSE Ratio } \\
\text { L/W }\end{array}$ \\
\hline 0.4 & L* & 0.41241 & 0.41407 & 0.033873 & 0.033870 & 0.94043 \\
& W & & 0.45876 & & 0.036016 & \\
\hline 2.0 & L & 0.00986 & 0.00954 & 0.001299 & 0.001299 & 0.99883 \\
& W & & 0.00859 & & 0.001300 & \\
\hline 3.6 & L & 0.00220 & 0.00203 & 0.000275 & 0.000275 & 1.00000 \\
& W & & 0.00167 & & 0.000275 & \\
\hline 5.2 & L & 0.00097 & 0.00069 & 0.000089 & 0.000089 & 1.00000 \\
& W & & 0.00053 & & 0.000089 & \\
\hline
\end{tabular}

\begin{tabular}{l|c|c|c|c|c|c}
\hline \multicolumn{7}{c}{ Matching modes of life distributions $(m=5.04, \xi=2, \mu=8, \nu=3)$} \\
\hline 0.4 & $\mathrm{~L}$ & 0.99473 & 0.99495 & 0.000699 & 0.000699 & 0.90633 \\
& $\mathrm{~W}$ & & 0.98622 & & 0.000772 & \\
\hline 2.0 & $\mathrm{~L}$ & 0.98147 & 0.98101 & 0.002631 & 0.002631 & 0.16675 \\
\hline & $\mathrm{W}$ & & 0.86682 & & 0.015776 & \\
\hline 3.6 & $\mathrm{~L}$ & 0.96977 & 0.97096 & 0.003891 & 0.003892 & 0.14554 \\
\hline 5.2 & $\mathrm{~W}$ & & 0.81860 & & 0.026743 & \\
& $\mathrm{~L}$ & 0.95873 & 0.95862 & 0.005628 & 0.005626 & 0.18409 \\
& $\mathrm{~W}$ & & 0.80082 & & 0.030563 & \\
\hline
\end{tabular}

${ }^{*} \mathrm{~L}$-Lognormal, W-Weibull. 
TABLE 3

Robustness of Bayes reliability estimator: True life distribution-lognormal $\left(m, \sigma^{2}\right)$, assumed life distribution-gamma $\left(\alpha^{*}, \beta\right)$, and inverted gamma $(\mu, \nu)$ prior distribution on scale parameter.

Sample size $=10, Q(R)=1$.

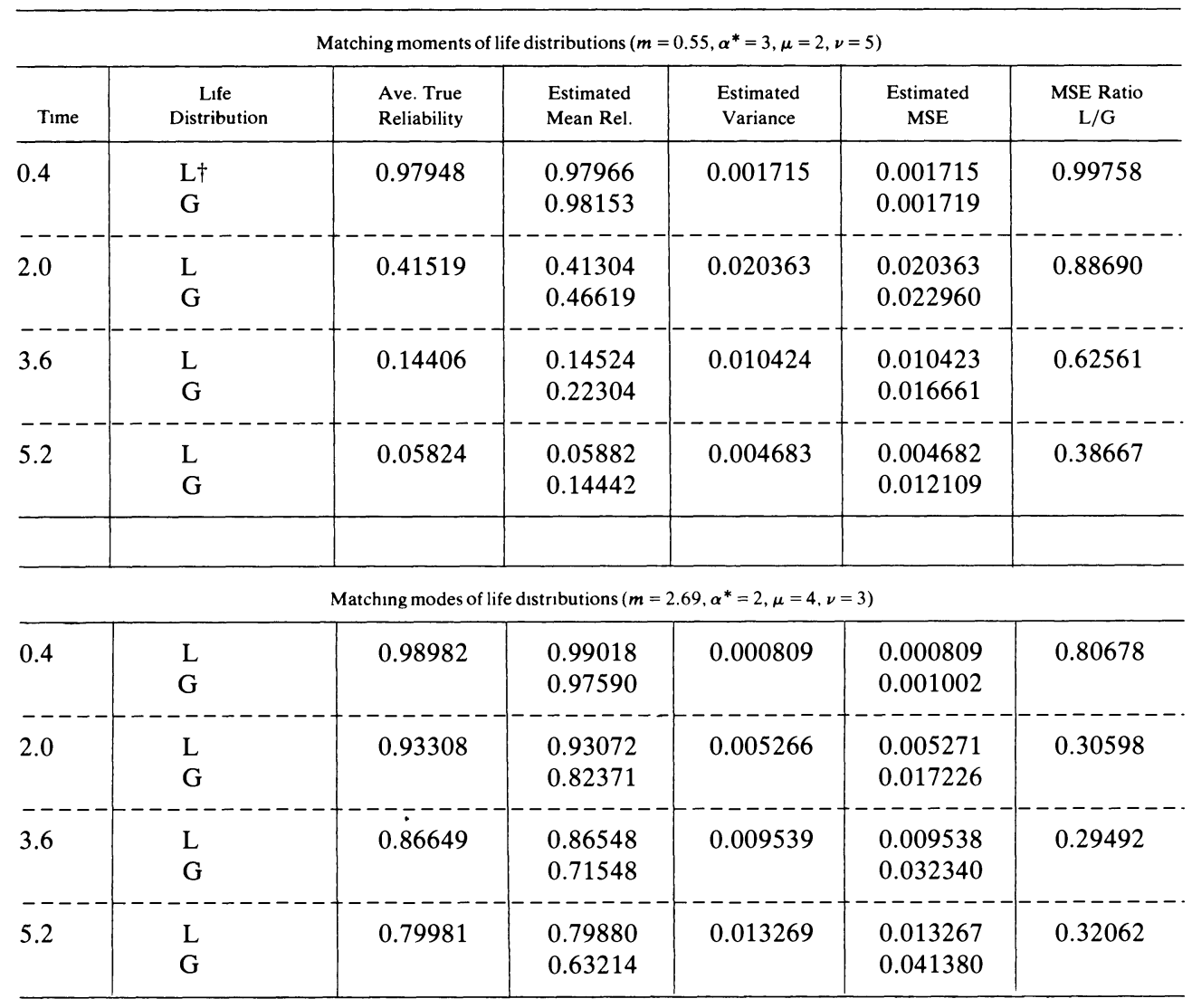

† L-Lognormal, G-Gamma.

5. Conclusions. The Bayes estimator $\hat{R}_{n}(t)$ of the reliability function for mixed failure time distributions is given by (3.4). The estimator is a function of both the prior or initial guess $F_{0}(t)$ of the unconditional (or mixed) failure time distribution $F(t)$ and the sample distribution function based on a random sample of $n$ lifetimes from $F(t)$. Even though $\hat{R}_{n}(t)$ converges almost surely uniformly in $t$ to the true unconditional reliability function $R(t)$ as $n \rightarrow \infty$, it was indicated by Monte Carlo simulations that the estimator is not robust with respect to all choices of $F_{0}$ even though the weight $p_{n}$ given to $F_{0}$ in the Bayes estimator was relatively small. This was true particularly for samples of size $n=5$ or $n=10$. Thus, even for this strongly consistent estimator the choice of the correct or nearly correct conditional failure time distribution is extremely important for both consumer and producer. However, the choice of the distribution $G$ of the random scale parameter of the Weibull distribution did not seem to affect the Bayes estimator very much even for small samples when the true and assumed $G$ have the same general fitting characteristics.

Acknowledgment. The authors are grateful to the referee for his helpful suggestions on improving this paper. 
TABLE 4

Robustness of Bayes reliability estimator: True life distribution-gamma $\left(\alpha^{*}, \beta\right)$, assumed life distribution-Weibull $(\xi, \theta)$, and inverted gamma $(\mu, \nu)$ prior distribution on scale parameter.

Sample size $=10, Q(R)=1$.

\begin{tabular}{|c|c|c|c|c|c|c|}
\hline \multicolumn{7}{|c|}{ Matching modes of life distributions $\left(\alpha^{*}=2, \xi=5, \mu=10, \nu=3\right.$ ) } \\
\hline Time & $\begin{array}{c}\text { Life } \\
\text { Distribution }\end{array}$ & $\begin{array}{l}\text { Ave. True } \\
\text { Reliability }\end{array}$ & $\begin{array}{l}\text { Estimated } \\
\text { Mean Rel. }\end{array}$ & $\begin{array}{l}\text { Estimated } \\
\text { Variance }\end{array}$ & $\begin{array}{l}\text { Estimated } \\
\text { MSE }\end{array}$ & $\begin{array}{l}\text { MSE Ratio } \\
\text { G/W }\end{array}$ \\
\hline 0.4 & $\begin{array}{l}\mathrm{G}^{\dagger} \\
\mathrm{W}\end{array}$ & 0.99157 & $\begin{array}{l}0.99127 \\
0.99176\end{array}$ & 0.000743 & $\begin{array}{l}0.000743 \\
0.000743\end{array}$ & 1.00000 \\
\hline 2.0 & $\begin{array}{l}\mathrm{G} \\
\mathrm{W}\end{array}$ & 0.86806 & $\begin{array}{l}0.86895 \\
0.79126\end{array}$ & 0.009411 & $\begin{array}{c}0.009410 \\
0.015307\end{array}$ & 0.61479 \\
\hline--- & - & ------ & ----- & ------ & -------- & ----- \\
\hline 3.6 & $\begin{array}{l}\mathrm{G} \\
\mathrm{W}\end{array}$ & 0.71324 & $\begin{array}{l}0.71475 \\
0.64991\end{array}$ & 0.017346 & $\begin{array}{l}0.017344 \\
0.021353\end{array}$ & 0.81229 \\
\hline 5.2 & $\begin{array}{l}\mathrm{G} \\
\mathrm{W}\end{array}$ & 0.57700 & $\begin{array}{l}0.57842 \\
0.52596\end{array}$ & 0.019624 & $\begin{array}{l}0.019622 \\
0.022225\end{array}$ & 0.88289 \\
\hline
\end{tabular}

† G-Gamma, W-Weibull.

TABLE 5

Robustness of Bayes reliability estimator for Weibull life distribution with respect to the prior distribution of scale parameter.

True prior: Inverted gamma $(40,3)$.

Assumed prior: General uniform (.008, .158).

Sample size $=5, Q(R)=1$.

\begin{tabular}{|c|c|c|c|c|c|c|c|}
\hline & Time & Prior & $\begin{array}{l}\text { Ave. True } \\
\text { Reliability }\end{array}$ & $\begin{array}{l}\text { Estimated } \\
\text { Mean Rel. }\end{array}$ & $\begin{array}{l}\text { Estimated } \\
\text { Variance }\end{array}$ & $\begin{array}{l}\text { Estimated } \\
\text { MSE }\end{array}$ & $\begin{array}{c}\text { MSE Ratio } \\
\text { (True/Assumed) }\end{array}$ \\
\hline \multirow{4}{*}{$\xi=1$} & 0.4 & $\begin{array}{l}\mathrm{IG}^{*} \\
\mathrm{GU}\end{array}$ & 0.97059 & $\begin{array}{l}0.97080 \\
0.97030\end{array}$ & 0.003983 & $\begin{array}{l}0.0039818 \\
0.0039818\end{array}$ & 1.00000 \\
\hline & 2.0 & $\begin{array}{l}\text { IG } \\
\text { GU }\end{array}$ & 0.86384 & $\begin{array}{l}0.86441 \\
0.86222\end{array}$ & 0.016651 & $\begin{array}{l}0.016648 \\
0.016651\end{array}$ & 0.99986 \\
\hline & 3.6 & $\begin{array}{l}\mathrm{IG} \\
\mathrm{GU}\end{array}$ & $\begin{array}{l}------ \\
0.77218\end{array}$ & $\begin{array}{l}-1---- \\
0.76953 \\
0.76609\end{array}$ & 0.024399 & $\begin{array}{c}------ \\
0.024401 \\
0.024431\end{array}$ & $\begin{array}{c}0.99877 \\
0\end{array}$ \\
\hline & $\begin{array}{l}--- \\
5.2\end{array}$ & IG & 0.69305 & $\begin{array}{l}0.69724 \\
0.69290\end{array}$ & 0.029248 & $\begin{array}{c}------ \\
0.029260 \\
0.029242\end{array}$ & $\begin{array}{c}--------- \\
1.00060\end{array}$ \\
\hline \multirow{4}{*}{$\xi=4$} & 0.4 & $\begin{array}{l}\mathrm{IG} \\
\mathrm{GU}\end{array}$ & 0.99808 & $\begin{array}{l}0.99798 \\
0.99795\end{array}$ & 0.000280 & $\begin{array}{l}0.000280 \\
0.000280\end{array}$ & 1.00000 \\
\hline & $\begin{array}{l}--- \\
2.0\end{array}$ & $\begin{array}{l}\mathrm{IG} \\
\mathrm{GU}\end{array}$ & 0.36443 & $\begin{array}{r}----- \\
0.36304 \\
0.35806\end{array}$ & 0.032016 & $\begin{array}{l}------ \\
0.032011 \\
0.032050\end{array}$ & $\begin{array}{r}0.99879 \\
-------\end{array}$ \\
\hline & $\begin{array}{l}--- \\
3: 6\end{array}$ & $\begin{array}{l}\text { IG } \\
\text { GU }\end{array}$ & $\begin{array}{l}------ \\
0.00712\end{array}$ & $\begin{array}{r}------ \\
0.00735 \\
0.00793\end{array}$ & 0.001023 & $\begin{array}{l}0.001023 \\
0.001024\end{array}$ & $\begin{array}{c}--------- \\
0.99941\end{array}$ \\
\hline & $\begin{array}{l}--- \\
5.2\end{array}$ & $\begin{array}{l}--- \\
\text { IG } \\
\text { GU }\end{array}$ & 0.00014 & $\begin{array}{r}----- \\
0.00019 \\
0.00017\end{array}$ & 0.000028 & $\begin{array}{l}------ \\
0.000028 \\
0.000028\end{array}$ & $\begin{array}{r}1.00000 \\
1 .-----\end{array}$ \\
\hline
\end{tabular}

* IG-Inverted Gamma, GU-General Uniform. 
TABLE 6

Robustness of Bayes reliability estimator for Weibull life distribution with respect to the prior distribution of scale parameter.

True prior: General uniform $(.008, .158)$.

Assumed prior: Inverted gamma $(40,3)$.

Sample size $=10, Q(R)=10$.

\begin{tabular}{|c|c|c|c|c|c|c|}
\hline & Time & Prior & $\begin{array}{l}\text { Ave. True } \\
\text { Reliability }\end{array}$ & $\begin{array}{l}\text { Estimated } \\
\text { Mean Rel. }\end{array}$ & $\begin{array}{l}\text { Estimated } \\
\text { Variance }\end{array}$ & $\begin{array}{c}\text { MSE Ratio } \\
\text { (True/Assumed) }\end{array}$ \\
\hline \multirow{4}{*}{$\xi=1$} & 0.4 & $\begin{array}{l}\mathrm{GU}^{*} \\
\mathrm{IG}\end{array}$ & 0.96761 & $\begin{array}{l}0.96729 \\
0.96878\end{array}$ & 0.00079 & 0.99837 \\
\hline & 2.0 & $\begin{array}{l}\text { GU } \\
\text { IG }\end{array}$ & 0.85072 & $\begin{array}{l}0.85011 \\
0.85667\end{array}$ & 0.00318 & 0.98913 \\
\hline & $\begin{array}{l}--- \\
3.6\end{array}$ & $\begin{array}{l}\text { GU } \\
\text { IG }\end{array}$ & 0.75152 & $\begin{array}{l}0.75091 \\
0.76124\end{array}$ & 0.00477 & $\begin{array}{c}0.98064 \\
0\end{array}$ \\
\hline & 5.2 & $\begin{array}{l}\text { GU } \\
\text { IG }\end{array}$ & 0.66701 & $\begin{array}{l}0.66662 \\
0.67964\end{array}$ & 0.00545 & 0.97162 \\
\hline \multirow{4}{*}{$\xi=4$} & 0.4 & $\begin{array}{l}\text { GU } \\
\mathrm{IG}\end{array}$ & 0.99789 & $\begin{array}{l}0.99786 \\
0.99796\end{array}$ & 0.00005 & 0.99990 \\
\hline & 2.0 & $\begin{array}{l}\mathrm{GU} \\
\mathrm{IG}\end{array}$ & 0.33456 & $\begin{array}{l}0.33509 \\
0.35003\end{array}$ & 0.00568 & 0.95960 \\
\hline & 3.6 & $\begin{array}{l}\text { GU } \\
\text { IG }\end{array}$ & 0.01056 & $\begin{array}{l}0.01057 \\
0.00885\end{array}$ & $\begin{array}{l}----- \\
0.00027\end{array}$ & $\begin{array}{c}0.98922 \\
-------\end{array}$ \\
\hline & 5.2 & $\begin{array}{l}\text { GU } \\
\text { IG }\end{array}$ & 0.00003 & $\begin{array}{l}0.00002 \\
0.00008\end{array}$ & $0.5 \times 10^{-6}$ & 0.99477 \\
\hline
\end{tabular}

* GU-General Uniform, IG-Inverted Gamma.

\section{REFERENCES}

[1] G. E. P. Box AND G. TIAo, Bayesian Inference in Statistical Analysis, Addison-Wesley, Reading, MA, 1973.

[2] George C. CAnavos And Chris P. Tsokos, Bayesian estimation of life parameters in the Weibull distribution, Operations Res. 21 (1973), pp. 755-763.

[3] Thomas S. Ferguson, A Bayesian analysis of some nonparametric problems, Ann. Statist., 1 (1973), pp. 209-230.

[4] - Prior distributions on spaces of probability measures, Ibid., 2 (1974), pp. 615-629.

[5] C. M. HARRIS AND N. D. SINGPURWALLA, Life distributions derived from stochastic hazard rates, IEEE Trans. Reliability, R-17 (1968), pp. 70-79.

[6] J. J. HIGgINS AND C. P. Tsokos, On the behavior of some quantities used in Bayesian reliability demonstration tests, to appear.

[7] E. S. Pearson And H. O. Hartley, Eds., Biometrika Tables for Statisticians, Vol. 2, Cambridge University Press, Cambridge, 1972.

[8] CHRIS P. Tsokos, Bayesian approach to reliability using the Weibull distribution with unknown parameters and its computer simulation, Rep. Statist. Appl. Res. Un. Japan Sci. Engrs., (1972), pp. 123-134.

[9] S. S. WiLks, Mathematical Statistics, John Wiley, New York, 1962. 